\title{
AVALIAÇÃO DE SOFTWARE COMERCIAL PARA SIMULAÇÃO CFD DE MACROSSEGREGAÇÃO.
}

\author{
A. L. NUNIS ${ }^{1}$, M. L. LIMA ${ }^{1}$ \\ ${ }^{1}$ Instituto de Pesquisas Tecnológicas do Estado de São Paulo, Laboratório de Processos \\ Metalúrgicos. \\ E-mail para contato: alnunis@ipt.br
}

\begin{abstract}
RESUMO - Esse trabalho teve como objetivo avaliar o módulo de solidificação do software comercial FLUENT para simulação de macrossegregação durante a solidificação de ligas metálicas. A avaliação foi feita por comparação a um algoritmo próprio desenvolvido e validado com dados experimentais e de literatura. Os resultados mostram que o módulo de solidificação do software comercial é falho para boa representatividade do fenômeno de macrossegregação e se limita a condições específicas de solidificação como, por exemplo, homogeneidade da fração de soluto no líquido.
\end{abstract}

\section{INTRODUÇÃO}

A macrossegregação é o fenômeno de variação de composição química na escala macroscópica (na escala das dimensões da peça) em peças que passam por uma etapa de solidificação. Essa variação de composição tem efeitos deletérios para algumas aplicações, como na produção de grandes lingotes, palhetas de turbinas ou lingotamento contínuo, por exemplo (Beckermann, 2002). Por outro lado, a macrossegregação de solutos é desejada em processos de refino por solidificação direcional como, por exemplo, no caso do refino de silício metalúrgico.

De maneira geral, devido à maior solubilidade de soluto no líquido, durante a solidificação de ligas metálicas ocorre a segregação de solutos na interface sólido-líquido na escala microscópica. Assim, a concentração de soluto no líquido aumenta com a progressão da solidificação (diminuição da fração de líquido). A macrossegregação ocorre quando há o transporte de soluto desse líquido com elevados teores de soluto para regiões de líquido com baixos teores na escala macroscópica (Beckermann, 2002). O transporte de soluto no líquido na escala macroscópica ocorre por mecanismos como difusão e convecção no líquido (Glicksman, 2011).

A modelagem de macrossegregação é antiga e se desenvolveu na década de 70 (Flemings, 1974) com resolução de modelos analíticos. Porém, com a evolução das técnicas computacionais, foi possível representar com maior precisão o fenômeno via o equacionamento do transporte das espécies entre as fases considerando a difusão e a convecção. A resolução desses problemas geralmente é apresentada na literatura pelo método dos volumes finitos (Voller e Brent, 1989; Lima, 2013, Li et al, 2014, entre outros).

O software comercial FLUENT, pertencente à empresa ANSYS, possui um módulo de solidificação e fundição que, se acionado em conjunto com o módulo de transporte de espécies, se propõe a representar a segregação de solutos durante a mudança de fase. Para isso, os desenvolvedores programaram no algoritmo do software a metodologia desenvolvida 


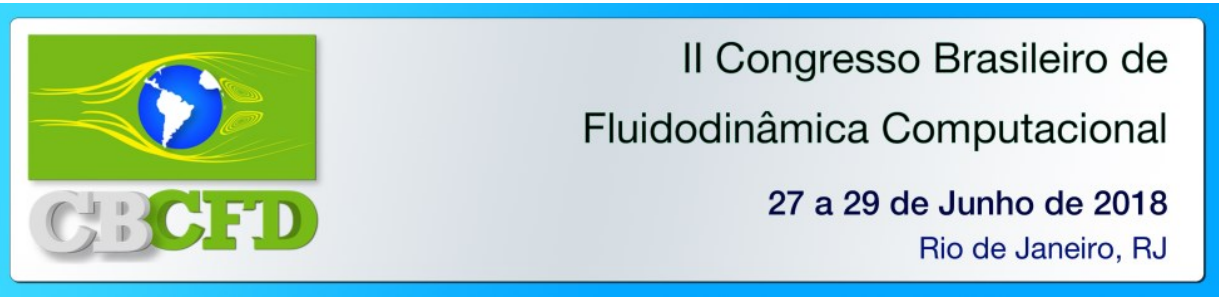

e publicada por Voller e Brent (1989) em que o módulo permite escolher entre o modelo para dendrita colunar com microestrutura dispersa (Equação 1) ou com microestrutura distinta (Equação 2).

$\frac{\partial}{\partial t}\left(\rho C_{m i x}\right)+\nabla\left(\rho u C_{l}\right)=\nabla\left(\rho_{S} g_{S} \mathrm{D}_{S} \nabla C_{S}+\rho_{l} g_{l} \mathrm{D}_{l} \nabla C_{l}\right)$
$\rho C_{\text {mix }}=\rho f_{l} C_{l}+\int_{0}^{g_{S}} C_{S} d \alpha$

Onde $C_{m i x}$ é a concentração da mistura (média volumétrica obtida em um volume microscópico definido), $g_{s}$ e $g_{l}$ são as frações volumétricas de sólido e líquido no volume de controle, $f_{l}$ é a fração mássica de líquido e $\alpha$ é uma variável falsa para integração.

Nesse trabalho, os autores testaram para diferentes condições as capacidades e limitações do módulo do FLUENT. Essa avaliação foi feita por comparação ao algoritmo desenvolvido e validado com dados experimentais e de literatura por Lima (2013) e publicados por Lima et al.(2017).

\section{METODOLOGIA}

Para realizar as atividades desse trabalho, foi utilizado o software comercial FLUENT 13.0. Contudo, até a versão 18.2 o módulo de solidificação do FLUENT não passou por alterações significativas. O caso aqui estudado foi a solidificação direcional da liga Si$0,2 \% \mathrm{Fe}$, as condições de contorno e as propriedades são apresentadas na Tabela 1 .

Tabela 1 - Propriedades para liga $\mathrm{Si}-0,2 \% \mathrm{Fe}$.

\begin{tabular}{l|c}
\hline Difusividade mássica no líquido $\left[\mathbf{m}^{2} / \mathbf{s}\right]$ & $1,8 \times 10^{-8}$ \\
\hline Calor latente de solvente puro $[\mathbf{J} / \mathbf{k g}]$ & 1.782 .000 \\
\hline Calor específico $\boldsymbol{C} \boldsymbol{p}\left[\mathbf{J} /\left(\mathbf{k g}{ }^{\circ} \mathbf{C}\right)\right]$ & 968 \\
\hline Condutividade térmica $[\mathbf{W} /(\mathbf{m K})]$ & $22,49+0,025262 * \mathrm{~T}[\mathrm{~K}]$ \\
\hline Densidade $\left[\mathbf{k g} / \mathbf{m}^{3}\right]$ & $2985,6-0,30105 * \mathrm{~T}[\mathrm{~K}]$ \\
\hline Temperatura liquidus $\boldsymbol{T}_{\boldsymbol{L}}[\mathbf{K}]$ e sólidus $\boldsymbol{T}_{\boldsymbol{s}}[\mathbf{K}]$ & $\mathrm{T}_{\mathrm{L}}=\mathrm{T}_{\mathrm{f}}+\sum \mathrm{m}_{\mathrm{i}} \mathrm{Y}_{\mathrm{i}} \mathrm{e} \mathrm{T}_{\mathrm{s}=} \mathrm{T}_{\mathrm{f}}+\sum \mathrm{m}_{\mathrm{i}} \mathrm{Y}_{\mathrm{i}} / \mathrm{K}_{\mathrm{i}}$ \\
\hline Temperatura de fusão de solvente puro $\boldsymbol{T}_{\boldsymbol{f}}[\mathbf{K}]$ & 1687,15 \\
\hline Temperatura de eutético $\boldsymbol{T}_{\boldsymbol{e}}[\mathrm{K}]$ & 1475,15 \\
\hline Coeficiente angular da linha liquidus $\boldsymbol{m}[\mathbf{K}]$ & $-500,3$ \\
\hline Coeficiente de Partição $\boldsymbol{K}_{\boldsymbol{i}}$ & $8 \times 10^{-6}$ \\
\hline Fração mássica de soluto no eutético $\boldsymbol{y}_{\boldsymbol{i}}$ & 0,423 \\
\hline
\end{tabular}

Como condições de contorno foram utilizadas as curvas de temperatura em função do tempo obtidas experimentalmente obtidas por Lima et al. (2017). A malha utilizada foi constituída de elementos de $0,1 \mathrm{~mm}$ de comprimento. A temperatura inicial é de $1687,2 \mathrm{~K}$ e a concentração inicial de Fe é $0,2 \%$ em massa. No presente trabalho duas condições foram avaliadas: a primeira considerou a solidificação direcional sem convecção e a segunda 


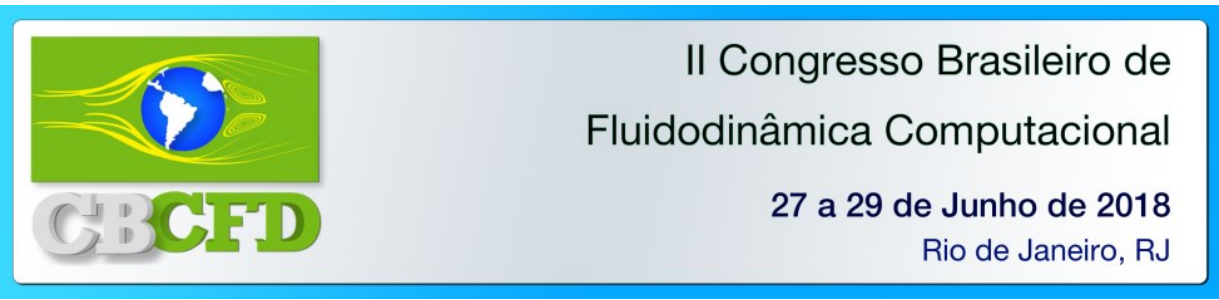

considerou a solidificação com convecção forçada no liquido. Para gerar essa convecção foi considerada a injeção de gás no líquido e utilizado o modelo de turbulência escolhido foi o k$\mathrm{kl}-\omega$.

\section{RESULTADOS}

As Figuras 1 e 2 apresentam os resultados de evolução da fração de líquido (interface de solidificação) e do perfil de fração de soluto em diferentes instantes para as duas condições simuladas: sem convecção (Figura 1) e com convecção forçada (Figura 2).
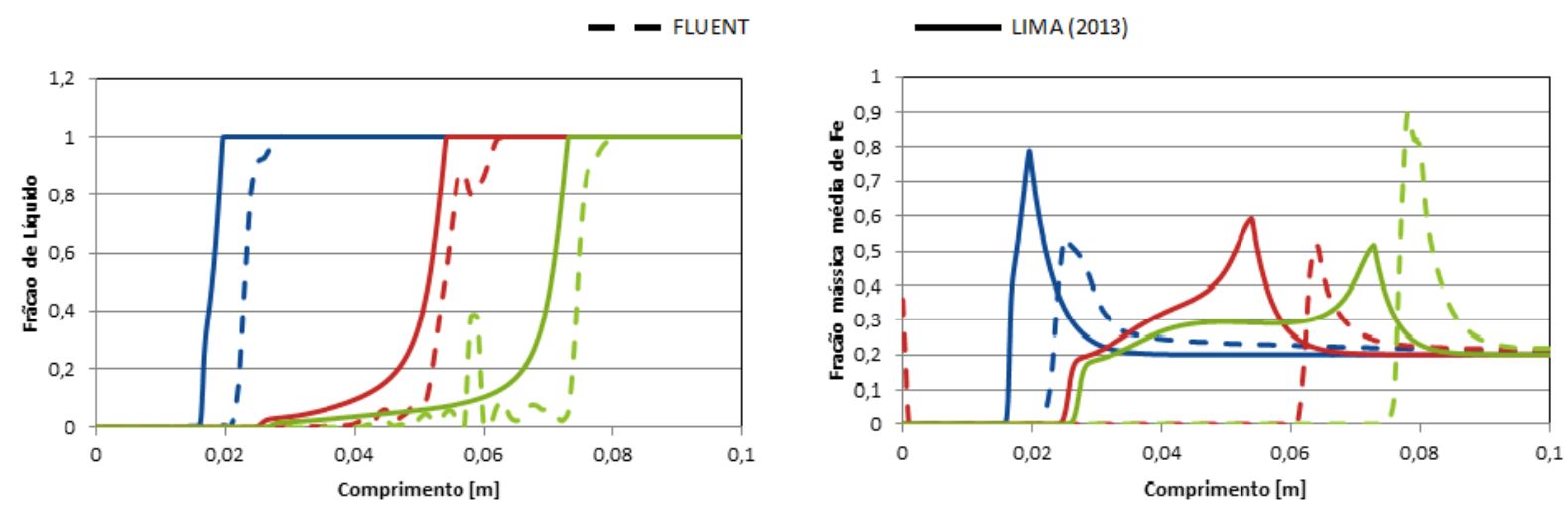

Figura 1 - Evolução de fração de líquido e fração média de soluto para os tempos de 10,7 ks (azul), 17,2 ks (vermelho) e 20,2 ks (verde) sem agitação forçada.
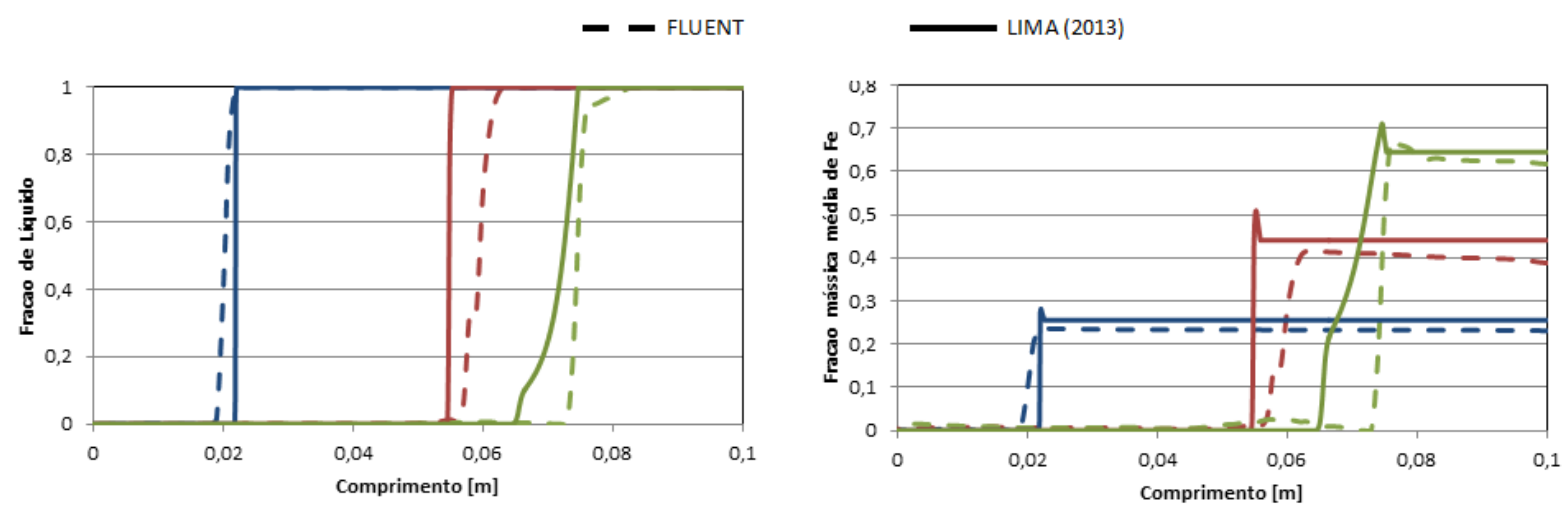

Figura 2 - Evolução de fração de líquido e fração média de soluto para os tempos de 10,7 ks (azul), 17,2 ks (vermelho) e 20,2 ks (verde) com agitação forçada.

Para o caso sem convecção (Figura 1) os resultados obtidos com o FLUENT e o com o modelo desenvolvido por Lima (2013) apresentam diferenças significativas. A frente de solidificação calculada pelo modelo do FLUENT é pouco mais rápida, ou seja, está em uma posição mais avançada para um dado instante quando comparada à posição da frente obtida com o modelo de Lima (2013). Nessa condição (sem convecção), os perfis de fração de soluto obtidos pelos dois modelos também são significativamente diferentes. .

A transição entre as regiões totalmente sólido e totalmente líquido, ou seja, a região de coexistência de sólido e liquido é denominada de zona pastosa. A diferença entre os 


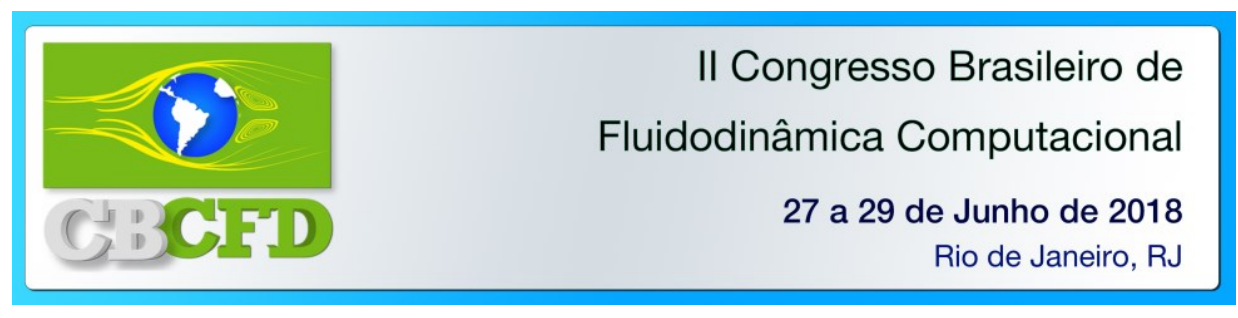

resultados dos modelos observadas na Figura 1 para na formação da zona pastosa (fração de líquido) pode ter origem no modo em que os balanços de massa e energia são realizados nas fases. O modelo de Lima (2013) aplicou os balanços na interface das fases enquanto que o modelo de mistura para a condição Lever do FLUENT considera o balanço como uma média no volume de controle assim como a temperatura da mistura para cálculo da temperatura liquidus.

As diferenças entre os dois modelos diminuem para o caso em que há convecção forçada no líquido (Figura 2). A convecção forçada aumenta a homogeneidade de soluto e os resultados mostram uma diminuição na extensão da zona pastosa e uma aproximação dos resultados entre os modelos. Esses resultados indicam que o módulo de solidificação do FLUENT tem melhor desempenho quando formação da zona pastosa não é significativa. Possivelmente, as diferenças entre o modelo do FLUENT e o modelo de referência podem ser reduzidas alterando o modo como os balanços de massa e energia são realizados no FLUENT implementando a solidificação no FLUENT via equações definidas pelo usuário (UDF). Esse trabalho está em desenvolvimento.

\section{CONCLUSÕES}

Nesse trabalho foi possível demonstrar que o módulo de solidificação do FLUENT possui limitações quando utilizado para avaliar a macrossegregação durante a solidificação de ligas. Para condições de solidificação que levam à formação de uma zona pastosa extensa, o erro no cálculo do perfil de soluto e fração de líquido é significativo. Esse erro diminui para casos em que a zona pastosa é menos extensa.

\section{REFERENCIAS}

BECKERMANN, C.; Modelling of macrosegregation: Aplications and future needs. International Materials Reviews. Vol 47 (5), pg. 243-261. 2002

GLICKSMAN, M. E.; Principles of Solidification. Springer, 2011.

LI, J.; WU, M.; LUDWIG, A.; KHARICHA, A.; Simulation of macrosegregation in a 2,45ton steel ingot using a three-phase mixed columnar-equiaxed model. International Journal of Heat and Mass Transfer. Vol 72, pg. 668-679. 2014

LIMA, M. L.; Refino de silício metalúrgico por solidificação direcional transiente. Dissertação (Mestrado em Ciências). Escola Politécnica - Universidade de São Paulo. São Paulo, 2013.

LIMA, M. L.; MARTORANO, M. A.; FERREIRA NETO, J. B.; Macrosegregation of impurities in a metallurgical silicon ingot after transient directional solidification. Materials Research. Vol 20, n 4, pg. 1129-1135. 2017

VOLLER, V. R.; BRENT, A. D.; The modelling of heat, mass and solute transport in solidification systems. International Journal of Heat and Mass Transfer. Vol 32(9).pg 17191731. 1989 